\title{
Método dos Mínimos Quadrados aplicado em Filogenia
}

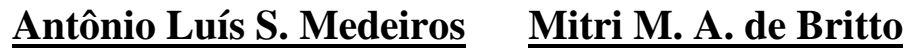 \\ Seção de Ensino Básico SE/1, IME \\ 22290-270, Rio de Janeiro, RJ \\ E-mail: aluis.852@gmail.com, britto.mitri@gmail.com
}

\author{
Nelson Antônio Borges Garcia \\ Seção de Ensino Básico SE/1, IME \\ 22290-270, Rio de Janeiro, RJ \\ E-mail: nborgesster@gmail.com
}

\section{RESUMO}

Em muitas aplicações há a necessidade de se determinar uma solução aproximada para um sistema linear mesmo sem solução. Usando o Método dos Mínimos Quadrados isso torna-se possível.

Neste artigo é estudada a utilização do método dos Mínimos Quadrados aplicado em Matrizes de Distâncias.

O objetivo deste trabalho é estudar métodos de reconstrução de árvores filogenéticas e estudar aplicações do método dos mínimos quadrados em problemas desse tipo, de forma a compreender a vantagem de sua utilização na filogenia. Para tanto, três métodos distintos para a construção de árvores filogenéticas serão utilizados a partir da mesma matriz de distâncias, com algumas espécies de animais, de forma que, após a aplicação dos métodos, os resultados possam ser comparados no intuito de aferir a aplicabilidade de cada um.

Inicialmente introduzidos por Cavalli-Sforza e Edwards[1], métodos de matrizes de distâncias utilizando Mínimos Quadrados foram sendo desenvolvidos e modificados durante os anos. O método de inferência de árvores por mínimos quadrados apresentado é um dos mais aceitos e bem justificados estatisticamente. $\mathrm{O}$ critério básico para aferir a qualidade da árvore aproximada (inferida) é dado pelo valor da soma dos quadrados dos resíduos (diferenças entre valores das distâncias reais e aproximadas) e é usado o algoritmo proposto por Albrecht et al[3], o qual consiste em uma sequência de passos que distribui a criação de árvores filogenéticas em um grupo de processos, onde a cada iteração identificam-se as melhores árvores, que são mantidas. Este algoritmo baseado em Falsenstein[2] é estudado neste trabalho, e na Figura 1 abaixo, pode-se ver o resultado obtido para uma determinada matriz de distâncias.

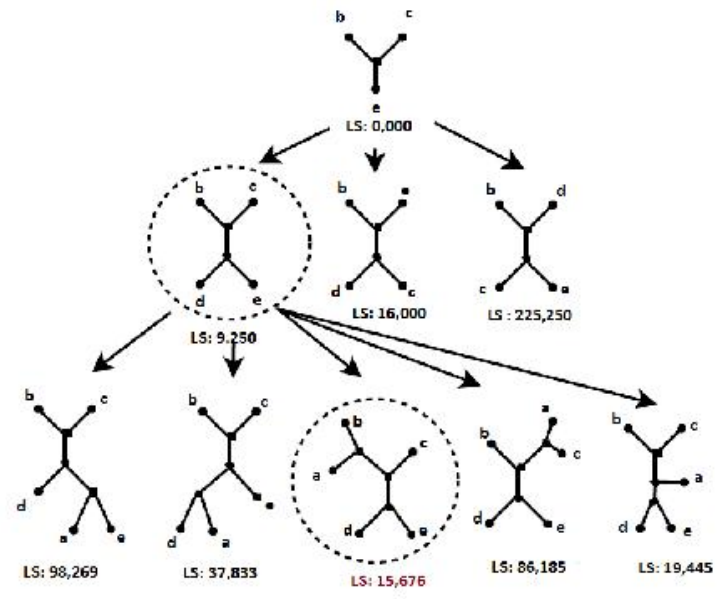

Figura 1: Exemplo de obtenção da árvore ótima com o Método dos Mínimos Quadrados.

Palavras-chave: Árvores Filogenéticas, Mínimos Quadrados, Matrizes de Distâncias 


\section{Referências}

[1] Cavalli-Sforza, L.L. and Edwards, A.W. Phylogenetic analysis: models and estimation procedures. Am J Hum Genet 19: 233-257. 1967.

[2] Felsenstein, Joseph. Inferring phylogenies. Massachusetts: Sinauer Associates, 2003. 580p.

[3] Albrecht, F.F. and Hubner, J.F. and Davilla, A. A Distributed Algorithm for Phylogenetics Inference. BSB2007 Poster Proceedings, 2007 